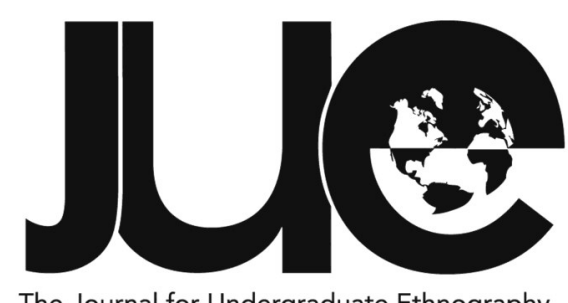

\title{
"There's A Little Bit of That Magic Where I'm Becoming Something Else": LGBT+ Furry Identity Formation and Belonging Online
}

Mary Heinz

Goucher College, heinzmarye@gmail.com

\section{ABSTRACT}

Active and open identification with animals and the creation of anthropomorphic (or zoomorphic) fursonas have become infamous on the internet, yet published research on the subculture is lacking. This ethnographic study explores this under-examined subculture by considering how individuals who identify as LGBT+ and as furry find and experience community online in ways that contribute to feelings of belonging, inclusion, and overall well-being. Through a series of semi-structured interviews, it was found that participating LGBT+ furries experienced an increase in self-reported emotional well-being when allowed to engage with online furry fandom. Given that furry identity is inherently non-normative, the fandom becomes an accepting space for other non-normative identities, like LGBT+ identities. By creating accepting online communities, those without access to supportive communities in their off-line lives can learn about and explore non-normative identity without judgement. These spaces may allow for the accumulation of multiple non-normative identities, all of which are in relative harmony within online furry fandom, which serves as a "catch-all" identity. Existing within this space had positive affects on the well-being of the participants. 
n 1993, Peter Steiner illustrated a comic featuring two dogs for The New Yorker. one is on the computer while the other looks on. The first dog says to the other, "On the internet, nobody knows you're a dog" (Steiner 1993; see also Wikipedia, 2020). At that time, the anonymity of the internet was alluring and presented users with the opportunity to be other people or to play with their identity (Boellstorff 2008). Today, in 2019, the opposite has become true. According to some studies, the internet is no longer as seductively anonymous as it once was, as contemporary users of social media are more likely to present their in real life (IRL) selves instead of constructing entirely new identities (Davis and Weinstein 2017).

If you identify as or with dogs on the internet today, everyone seems to know about it. Active and open identification with animals and the creation of anthropomorphic (or zoomorphic) fursonas have become infamous on the internet. Anthropomorphism indicates the application of human characteristics to animals, whereas zoomorphism is the application of animal characteristics to humans (Gerbasi et al. 2008). Within the literature on furry identity and identity performance, the terms appear to be used interchangeably, with anthropomorphism used more frequently. For the sake of this paper, anthropomorphism will be used to describe both phenomena.

Furries and non-furries alike enjoy making jokes and memes at the expense of furry fandom, a prominent internet and IRL subculture. Yet, infamy does not lead to understanding. Popular conceptualizations of furries often paint them as mentally ill sexual deviants (Gerbasi et al. 2008, 199). While sexuality can be a part of one's experience with furry identity (Strike 2017), perceptions of the fandom by outsiders tend to frame furry experience as exclusively erotic in nature (Jeannsone 2012, 5). This leads to backlash from non-furries in the form of bullying or exclusion. A notable example of maliciousness from nonfurries to furries occurred in 2014. Midwest Furfest, a large, annual furry convention in Chicago, was interrupted by an "intentional gas incident" that some consider to be a terrorist attack. Nineteen convention goers were hospitalized due to chlorine gas exposure. As reports of the attack spread, what followed suit was not horror but humor at the expense of the convention goers because they were furries. Additional claims were made that, because furries are considered deviant, they deserved the attack and bullying that followed. Victoria McNally (2014) writes extensively on the incident. Although this is an extreme example, it emphasizes how furries are viewed by mainstream society. Many furries also identify with the acronym LGBT+, which can exacerbate the backlash they experience since LGBT+ identities are also commonly considered deviant. In the context of this paper, the LGBT+ label refers to lesbians, gays, bisexuals, transgender people, as well as the many other nuanced individuals that find themselves identifying with communities and labels outside of heterosexual and/or cisgender identity.

Furry fandom is much more beyond stereotypical views. Joe Strike (2017), a selfidentified furry, defines a furry as anyone with an above-average interest in anthropomorphic characters, even if they do not self-identify with the term. The few academics who have pursued the study of furry subculture, however, consider furries to be those who strongly identify with, or view themselves as, an animal or other nonhuman creature and use the term to label themselves (Gerbasi et al. 2008, 198; Jeansonne 2012, 73; Půtová 2013, 246). For the sake of this study, I will use the academic definition of a furry: one who actively identifies with the term. Furries engage in the creation of art and music, participate in video gaming and dance competitions, and organize events ranging from several thousand visitors at a convention to ten friends at a bowling alley. Furry fandom has a thriving physical and virtual culture that involves more than what is perceived to be sexual deviance by outsiders. 
Identifying as a furry can lead to the creation of a fursona, or an anthropomorphic character depicting the real or constructed personality of the maker (Jeansonne 2012, ix). Fursonas are made manifest through artwork shared in online spaces or fursuits-a handcrafted suit allowing the wearer to become their fursona in physical reality (Gerbasi et al 2008; Jeansonne 2012). In this way, a furry may experience a selfhood that is composed of dual identities or identifications. This, and the way furry fandom functions as an online community, is not widely explored by the available literature. Studies tend to focus on the physical embodiment of furry identity through fursuiting, instead of on the formation of identity and influences of the online furry fandom community on well-being. It is important to distinguish the embodied experience of identity and physical community from the performance of identity and online communities for several reasons. There is a notable cost barrier for physical embodiment. Fursuits, full or partial, can cost thousands of dollars, making it difficult to access that means of identity embodiment. A wider range of people can express their identities online because of the lower cost of entry to the community, making the scene more diverse in terms of socio-economic status and racial identity. The internet, as explored in the study presented herein, is also a place where individuals learn to accept and be themselves through access to information and other individuals who are socially deviant. This further contributes to the diversity of the fandom online, as individuals from all over the world can be exposed to the fandom and learn about it without physical exposure. Regional, racial, and socio-economic factors influence identity, furry or not, but these factors are not explored within the previous literature on furries. Past ethnographers attended conventions and studied fursuiters. Due to the cost of entry to the convention and fursuiting as a practice, those studied in past literature were, in general, white men (Gerbasi et al. 2008). It should also be noted that expression and experience of identity and community online may be inherently different from real life because of the medium, but this has not yet been explored within the field of furry studies.
Additionally, the ways in which furry identity intersects with other identities, like LGBT+ identities, and how that affects belonging and well-being is unexplored by the available literature. The following study aims to expand academic understanding of furry identity and how it intersects with LGBT+ identities through an exploration of the ways in which these identities are formed online and how they affect well-being and belonging. Based on the findings of this study, I assert that furry fandom, because of its role in the lives of participants as a positive and accepting space, grants those with socially deviant identities a place to belong and thrive.

\section{What Do You Mean "Furry"?}

The fandom gained traction as a North American subculture in the 1980s when the "founding" members of furry fandom began meeting at conventions intended for science fiction media enthusiasts (Strike 2017). Anthropomorphism is, however, a much older practice. Půtová (2013) compares contemporary furries, in their physical embodiment and online presentation of identity, to anthropomorphic depictions of sorcerers in cave paintings, some dating back to 30,000 BCE. She notes how both furries and sorcerers have the ability to transcend reality and assume multiple identities through their animal self or selves (Půtová 2013, 247). Although what transcendence means for the individual experiencing it is not defined in the article, Pütová's (2013) work is still noteworthy because she is the only academic author in this literature review to reference the role of the internet in furry identity. She claims that the internet allows furries to create multifaceted alternative selves, which further allows transcendence from the "real" self to a different form of self (Pütová 2013, 247).

Similar to other fandoms, furry fandom online may provide members with a sense of community and, therefore, allow members to reap the benefits of community engagement. One study found that, in addition to participants experiencing stronger community bonds with their fandom than with their local, real life communities, their strong association with their fan interest was correlated with 
positive well-being (Chadborn, Reysen, and Edwards 2018, 247). For furries, much of the community they experience is online because conventions can be expensive and smaller meetups may be miles from home. While there is a common perception of internet communities and relationships as superficial or "not real" (Downing 2013), this is not necessarily the case for all online communities. Chadborn, Reysen, and Edwards $(2018,242)$ point out that communities are made up of four factors: a sense of belonging, integration, a shared emotional connection, and influence. These factors are all components of contemporary, online furry fandom.

Researchers agree that, because the internet is now highly integrated into our daily lives, its effects on tasks related to emotional and physical development, such as identity formation, have become an important avenue of exploration (Acosta et al. 2017; Downing 2013; Hatchel, Subrahmanyam, and Birkett 2017; Walks 2014). This study addresses a gap in the literature concerning furry fandom, which to date has focused on the physical manifestation of the fandom through fursuiting at conventions (Carlson 2011; Gerbasi et al. 2008; Jeansonne 2012). The importance and prominence of online communities that exist on websites like DeviantArt, FurAffinity, and Twitter is largely ignored. Thus, this study contributes to both furry studies and research concerning online communities. In order to frame my research on furry identity and community, I will examine how other online communities, including LGBT+ communities, have been analyzed with regards to online identity formation.

\section{Considering Identity Formation Online: Intersections of LGBT+ and Furry Identity}

Through fandom spaces, members are allowed to express and explore other aspects of selfidentity that may be stigmatized outside of the fandom, including LGBT+ identities (Chadborn, Reysen, and Edwards 2018, 243). Due to this, furry fandom and the LGBT+ community are inherently linked. As a group that deviates from social norms, furry fandom tends to be more accepting of other identities labeled as deviant or non-normative by mainstream societies; thus, a significant number of furries online are LGBT+ (Strike 2017). The internet allows both furries and LGBT+ individuals to establish social support networks where access to communities of like-minded individuals in real life is not possible (Acosta et al. 2017; Atay 2015; Downing 2013; Hatchel, Subrahmanyam, and Birkett 2017; Půtová 2013). Through communication with others of the same identity and interests, an individual is able to gain access to greater social support (Chadborn, Reysen, and Edwards 2018, 242), which can affect their well-being and identity formation.

Downing's (2013) study found that the community created by LGBT+ identities in online spaces became real community through the creation of highly valued relationships in online forums (51). The perceived value of these interactions therefore allows the internet to have significant effects on the development of those using it. One key aspect of identity development is the reciprocal nature of community involvement and identity formation. LGBT+ individuals share and gain selfknowledge by talking about their experiences and hearing the experiences of others, thereby asserting and forming their LGBT+ identity (Acosta et al. 2017, 91; Downing 2013, 48; Hatchel, Subrahmanyam, and Birkett 2017, 61). This reveals a dialogical aspect of identity creation and, potentially, selfhood in online spaces that will be further explained in the next section.

Studies within the field of sociology attribute the ability to share freely in this way to the anonymity of the internet (Acosta et al. 2017; Downing 2013). Davis and Weinstein (2017) argue that the increasing overlap of on- and offline life pressures LGBT+ people to be as consistent as possible across social media platforms (13). This contradicts the findings of similar studies which find it easy to compartmentalize one's role-identities and affiliate each with different social media platforms, blogs, or accounts on the same platform. Acosta et al. (2017), for example, found that many gay men participating in their study juggled multiple identities across internet platforms. This multiplicity is referenced with regards to furries by Pưtová (2013) and is 
similarly experienced through other mediums. Boellstroff's (2008) ethnography of an online role-playing game called Second Life found his participants reflecting on their possession of multiple identities, which he claimed were shaped largely by their perceived societal roles in the online game (119). This study implies that multiple identities can be accumulated online as users define the roles they want to have in their second lives, "unencumbered by social constraints or the particulars of physical embodiment" (Boellstroff 2008, 120). These identities, which are applied to and experienced through virtual characters, are developed using the same social tools as identities placed within the physical body (Tronstad 2008, 257). Within furry fandom, these multiple identities are similarly expressed through virtual characters that can be displayed through art or fursuits.

\section{Understanding Furries Using Dialogism and Identity Accumulation Theory}

Instead of relying on a view of a singular identity as the foundational core of a given person's selfhood, I take up the postmodernist school of identity theories where the self is composed of multiple and constantly changing identities (Brubaker and Cooper 2000, 8). As Půtová $(2013,247)$ notes in her analysis of furry fandom, furries often contain a multitude of identities that are created in the form of multiple fursonas and roles associated with the characterization of those fursonas. In addition to their identities as furries, however, individuals identify with multiple other roles that they serve within their communities, such as friend, co-worker, or deviant. The multiplicity within furry identities, as well as the multiplicity caused by the coexistence of at least two nonnormative identities (furry and LGBT+) within a single individual, can be described by the concept of multiple selfhoods.

The purpose of this study is to explore how individuals who identify as LGBT+ and as furry find and experience community online in ways that contribute to feelings of belonging and overall well-being. In order to do this, I am utilizing a combination of two theories of identity: dialogism and identity accumulation theory. Dialogism claims that an understanding of one's own self or culture is constructed through comparing oneself to an "other" who may be within one's cultural group or outside of it (Bandlamudi 1994, 463). In Bandlamudi's (1994) wording, this is the process that makes interpersonal events become intrapersonal and vice versa. This means that conversations, connections, or even witnessing another person outside of ourselves can create internal dialog about who we are, why we are, and whether or not to incorporate aspects of what we have learned into our sense of self. It also means that such an internal dialog can be turned outward to others through conversations, presentations of the self, and media, which, in turn, create internal dialog in others. This is a dialogical process in which the self both helps create and is created by the social and cultural worlds that surround it.

Dialogism is integrated within role-identity theory-a term used to describe identities related to the social position(s) one fills (Owens, Robinson, and Smith-Lovin 2010, 481). To explain, one can identify with the roles of mother, wife, daughter, friend, and co-worker all at once but only act within one or two of these roles at a time. Each have different niches to fill within our society and can have different linguistic and behavioral displays within a given individual. Similar to code-switching, an individual can move between these identities in order to better suit a given situation. Multiple role-identities has been found to positively affect well-being, as it can provide multiple avenues of support from those with similar role -identities and a sense that one's existence is meaningful because of the roles they serve (Lang and Lee 2005, 295). The same study found that multiple identities can contribute to a sense of existential security because an individual knows where they belong, but they can also cause internal and external conflict (Lang and Lee 2005, 295). For example, one's role as a co-worker could conflict with one's role as a parent through interpersonal events. These conflicts create intrapersonal dialogs between the role-identities that can reaffirm aspects of an identity and augment others.

This integration of dialogism and roleidentity theory leads to the concept of the dialogical-self: a selfhood in which a diversity of role-identities interact with each other and the 
outside world in a manner that synthesizes new and augmented identities and can affect the world around the self. It is a selfhood of constant change in which the "true self" is comprised of multiple moving parts, instead of one static, unchanging personality (Sampson 2008, 113-4; Van Meijl 2010). Viewing and interacting with others can lead to intrapersonal dialog about one's sense of self, leading to change in a given identity, and affecting the way an individual interacts with others and the world around them, thus continuing a cycle of creation.

For the participants of this study, these roleidentities could include their position as a furry, their position as LGBT+, their position as deviant, and more, as it is common for furries to create additional real and hypothetical roles for themselves in online spaces through their fursonas (Jeansonne 2012, xi). The second theory, identity accumulation theory, claims that having multiple identities can contribute to enhancing the quality of an individual's physical and emotional well-being in ways that are similar to the ones explained above for roleidentity theory (Lang and Lee 2005, 295; Owens, Robinson, and Smith-Lovin 2010, 482). Due to the multiplicity of identity that LGBT+ furries experience, they may have access to many online communities and reap the benefits of support from those communities. Multiple roleidentities may provide niche avenues of support for dealing with the consequences of a specific role-identity. Just as mothers can seek comfort and advice from other mothers but turn to their sisters to confide about their experience as daughters, LGBT+ furries can find support from non-furry LGBT+ individuals about their experiences with deviancy and LGBT+ identity. They can also talk to other furries and niche subgroups of the furry fandom about deviancy, fringe interests, and more. By having multiple role identities provided by their LGBT+ identity, their furry identity, and identities that may come to them through the expression of their fursona, LGBT+ furries may have a wider support network and, therefore, better well-being than those who may not similarly identify. This study, however, found that this was not the case for this group of participants due to the LGBT+ friendly nature of the furry fandom.

\section{Methodology}

For this study, I created an account on Twitter, a social media website and app, in order to establish a social media presence and rapport with potential informants. Twitter was selected for recruiting participants due to its popularity among furries to interact with each other and post art. Seven interviewees who identified as LGBT+ and furry were recruited. One was a personal friend. The others were strangers I contacted through Twitter direct messages with a brief summary of my position as a student researcher, the purpose of the study, and an invitation to participate. This research protocol was approved by the Institutional Review Board of Goucher College after completing the "Protecting Human Research Participants Online Training".

Three of the interviews were conducted using a semi-structured interview guide; one inperson and the others through Discord, an audio calling and chatting service favored by furries. These interviews were recorded and transcribed. The guide was designed to gauge how and why participants use the internet, how they came into their identities, who supported that transition, and how their identities and the communities they engage with online make them feel.

Four additional interviews were conducted using the same guide but in writing through Google Forums with additional follow-up through Twitter direct messages. While such an interview method can be considered unorthodox, I found it important to offer a variety of interview methods to potential participants. As a stranger to potential participants with little to no rapport between us, a request out of the blue for a spoken interview could be seen as frightening. As the researcher, I would have an audio recording of the informant's voice, which, for transgender people who are insecure with their voice, may be a daunting prospect and limit interest in the study. Additionally, anxiety related to phone conversations, especially with unfamiliar people, posed an issue. Through writing, a safe distance from the researcher and the informant is created, but valuable information is still gained. By offering this option, I made the study 
more accessible to people from a variety of backgrounds.

The interviewees range in age between 22 and 28. Four of the seven interviewees were white, one was Asian American, one was African American, and one self-identified as mixed. LGBT+ is an acronym used throughout this article as an abbreviated descriptor of the sexual and/or gender identities of all informants involved. None of the interviewees were heterosexual, identifying themselves as lesbian, asexual, bisexual, pansexual, or queer, and only two were cisgender while the others identified as non-binary or transmasculine. LGBT+ is used herein as an umbrella term as it is the most inclusive while also the least controversial within spaces that identify as lesbian, gay, bisexual, transgender, or any additional labels. To protect the participants, pseudonyms were chosen by the participants themselves or myself.

\section{Finding Space Where There Was None: Belonging Online}

The dialogical-self, which consists of multiple identities in dialog with one another and with the society outside of the self, is a means of creation. It creates our sense of self and can create new or edit identities within that self (Sampson 2008). It is also a means of creating the world around the self, or spaces within it in which to belong (Bandlamundi 1994). For all of the study participants, achieving a sense of belonging influenced the acquisition and acceptance of their non-normative identities. These spaces in which they found belonging were created through the expression of identity online by the LGBT+ furries that came before them. Logan (22), for example, said that they found furry fandom through DeviantArt, an art sharing website, at the height of what they refer to as "its sparkle dog phase." This was a reference to the prominence of anthropomorphic or furry influenced artwork and culture on DeviantArt in the form of brightly colored wolf or dog characters that were created by teenagers or young adults in the mid- to late-2000s. DeviantArt's platform, as well as other art platforms like FurAffinity, provided them with a playground in which they learned to create not only their own identity but also to create art.
However, non-normative identities are often stigmatized for deviating from mainstream culture. This stigma can be internalized and lead to self-denial and self-critiques. For the participants in this study, the experience of stigma stems primarily from deviating from sexuality that is perceived to be normal. For LGBT+ identities, this connection is clear. Any sexuality or gender identity outside of cisgender heterosexuality can break societal norms and may be persecuted. Furry identity can also be stigmatized, although not as commonly. Furries are not as widely known about within mainstream culture and, while a convention center was attacked, a heterosexual cisgender furry does not have a fear for their lives in the same way as LGBT+ individuals. While furry identity can be viewed as a stigmatized sexual kink or a form of bestiality to some, leading to harsh stereotypes, bullying, and exclusion, legislators are not (at the time of writing) creating anti-furry laws. The stigma that surrounds furry identity stems from the prevalence of erotic artworks of fursonas and the consensual sex between furries in fursuits that can be found and shared online among groups who seek to make fun or ridicule the subculture. This can force those who feel a connection to these deviant identities to deny them. Two informants expressed that they were exposed to stigma due to their desire to make art featuring anthropomorphic characters when they were children. Ray (22), for example, described their experience drawing anthropomorphic characters as a child and feeling stigmatized as a result:

Anything unfamiliar, or even anything seen as unpopular, in school is usually seen as lame, uncool, and/or something to be approached with scorn. For the most part, it was kids who weren't considered "nerds" who tended to approach any art I did as me being a furry. And the term furry carried such negativity in school that, even though I had no idea what it was and why they thought furry was wrong, I still vehemently said I wasn't one.

For Ray, childhood bullying led to the internalization of stigma and denial of furry identity for many years. This period of time was also marked by a lack of both self-esteem and 
supportive communities in real life for deviant identities. In order to conform to the communities and support networks that were available to the informants during their youth, they denied their deviant identities they began to cultivate that felt genuine and comfortable in favor of "normal" identities that kept them safe.

Lack of conformity can have starkly negative impacts on well-being and drive a wedge between an individual and the communities that they would otherwise have access to through other identities, like racial identity. Terra (24), for example, described at length what it is like to be African American and LGBT+. They considered their blackness to be the most important part of themselves and referred to the community created by racial identity as their core, foundational support group. They noted, however, that African American and LGBT+ identities "just don't get along," alluding to the homophobia and transphobia that is present in some African American communities. They explained further: “it's really hard to be black and be gay, or bi, or pan, or anything like that, because you kind of lose a lot of support from your core." The homophobia and transphobia that Terra experienced has separated them from spaces they could have belonged if not for their deviant identity. They currently live in a predominantly white town and only interact with one other African American regularly in real life.

It can be easier to deny a deviant identity in order to protect one's own physical and emotional safety. Yet it seems these "normal" identities fit uncomfortably on the participants and, sometimes after a period of many years, they pushed them aside to find communities in which more comfortable selves could be forged. For reasons they did not articulate, participants who initially denied non-normative identity still sought out non-normative community later in life. The pervasiveness of the internet within the day-to-day lives of the participants further exposed them to online furry fandom through art, videos, photographs, Twitter threads, or online friends. Large numbers of furries and LGBT+ people use Twitter to post about themselves, their interests, and their art. Based on the experiences of my participants, the act of knowledge sharing about the experience of deviant identity and what that identity is does not take place exclusively through direct conversation online through chat rooms, direct messages, or Twitter replies, which was the direct focus of Downing's (2013) research. Yet his concepts can still be applied here. What frequently takes place could be described as a form of witnessing positive representation by reading posts or viewing art and videos. Within his study, Downing (2013) determined that dialog between LGBT+ individuals about their deviant identities led to the individuals themselves gaining knowledge about themselves and others; these processes worked to affirm their identities as LGBT+. On contemporary social media websites, these kinds of conversations about deviant identities are not as common as what I refer to as "Show and Tell". While messaging is still common and part of the reason that the internet is important to several of the participants, it is overshadowed on Twitter by the creation of posts (text or image) that are then displayed on Twitter to be viewed and appreciated. The goal of these posts is not necessarily dialog; instead, the posts emphasize a want to Show (artwork, visual memes, etc.) or Tell (short tweets about one's day, Twitter essays on current events) something about a certain topic and share it with an audience for approval.

Show and Tell still functions as a means of gaining and sharing knowledge. Deviant creators produce content (artwork, videos, jokes, etc.) and others witness that content, gaining knowledge about the creator and their own identity through dialogical processes of identity creation or augmentation. Witnessing representation of the fandom or LGBT+ media encourages viewers to have internal dialogs about their role-identities. It enables those like Ray to challenge their denial and overcome it through exposure to deviant creators. Logan summarized the process by reflecting on their own experiences:

I was seeing people talk about it [gender and sexuality]. I was seeing people make jokes about it. I was seeing people poke fun and express it in a nonacademic way. The conversations around queerness, and gender fluidity, and all that. I was really thankful for it, because I think living in a highly gendered space was hard. And, I think 
understanding what dysphoria was gave me a lot of peace [...]. So it was relieving to know that there was something else.

Through their experience of belonging in online spaces, Logan's well-being increased. Logan did not have a phase of denial and has a small yet supportive community in real life. However, they also reflect on the loneliness of experiencing dysphoria in an all-girls' school where traditional presentation of womanhood was constantly enforced through casual language and treatment of the "ladies" who attended classes therein. This was relieved through knowledge sharing that took place in online spaces created through Show and Tell. The jokes and expression of gender in nonacademic ways were posts or conversations Logan witnessed, instead of conversations participated in by Logan themselves. Thereby, Logan was shown information and internalized it by others passively telling them about it.

In these spaces, the participants feel a sense of community with others who share a similar or same deviant identity. According to the participants, this sense of belonging has significant effects on their well-being. Shank (27), for example, reflects on how being his true self online has improved his self-esteem and the self-esteem of others:

I think a person is at their best when they feel free to express themselves genuinely and without inhibition. The way I identify is essentially me being true to myself allowing me to be more confident and secure in myself. When you're pretending to be someone you're not, I feel it often leads to self-doubt. There's that special little feeling you get when you say how you really feel out loud for the first time. Admitting something as simple as 'I like boys too' and meaning it feels really good.

Through furry fandom, he was able to freely express himself and his sexuality, which gave him greater confidence and better self-esteem.

With regards to well-being, it should be noted that within identity accumulation theory the identity that matters most to an individual is the most likely to have the greatest effect on one's well-being (Owens, Robinson, and SmithLovin 2010). When asked which one of their identities is the most important to them, some informants highlighted identities that made them feel the best, like their sexuality now that they are free to express it as they please. Others, paradoxically, highlighted identities that affected them the most negatively. Ray says the identity they consider to be the most important is their trans-masculine identity because of the frequent "face-to-face backlash" they experience daily. In response to me encouraging them to reflect on an identity that affects them positively, they doubled down on their initial answer. Despite the struggles, being out as a transgender person has improved their overall well-being. They now struggle less with self-doubt and body image issues because engagement with online communities helped them to accept this identity.

All is not perfect on the internet, however. Accepting and validating communities are not without their problems. Some of the informants did mention the existence of pedophiles, Nazis, incest advocates, or other problematic individuals within their fandom that give furry fandom a bad name outside of the community. Fandom members do take it upon themselves to police who can and cannot be within their fandom, actively trying to run out undesirables and their supporters through harassment, memes, and "cancel culture," or the process through which a community decides it is no longer ethical to follow or like a social media personality. Some consider this to be a good thing, but others find it treacherous. Terra refers to this pattern as "furry drama," but does not want to take the value of the work lightly. They do, however, express an intense desire to avoid "furry drama", a feeling that is mirrored by other participants in the study. However, avoiding this aspect of the fandom can be difficult if one has significant social capitalwhich comes in the form of followers, in this case. Terra describes posting potentially controversial statements or media on Twitter as, "kind of like, I pull the pin on the grenade and I throw it out, and then I'm just like, 'Okay, I can go back somewhere else,"' indicating that they, effectively, flee the scene to avoid drama or excessive attention associated with the post. Such posts usually involve a statement of opinion, usually concerning politics, or dislike of other fandom members or notable figures 
online. This is followed by extensive discourse within the comments, ranging from blind support to aggressive dissent.

Terra was not able to verbally explain why they engage in Show and Tell despite the fear of backlash from their own community in real life. They instead remark, "I guess it's just the social media feedback loop. You see a ton, a whole bunch of people just liking your stuff and you're like, 'That's cool. I can do that again.' And You do." This may reflect a desire to feel a part of and contribute to the community, even if doing so puts oneself at risk of becoming "canceled".

As referenced in the introduction, online presentation of the self is more commonly in line with presentation of the self in real life (Davis and Weinstein 2017). Who the participants are online is, generally, who they are offline. After acquiring their deviant identities, they made no mention of intentionally hiding their identities from others in real life or the benefits of anonymity in online spaces. It appears that, for the participants in this study, being in online furry communities allows people to be themselves through their various role identities without feeling shame, both on and offline. The online self is fully integrated with the IRL self. Rubi (28) comments on this phenomenon by saying, "it's like you used to be able to go online and be something else, or whatever. Like you could have an alias, and now it's almost like you are the alias." He explains that this is caused by the omnipresence of the internet in our day-to-day lives, which falls in line with Davis and Weinstein's (2017) study. Through social media, the online self and the IRL self become one and the same. The infamous anonymity of the internet is lost. This finding is of note due to the emphasis on anonymity in past literature concerning identity formation of young LGBT+ people online (Acosta et al. 2017; Downing 2013; Hatchel, Subrahmanyam, and Birkett 2017).

For the participants in this study, the integration of these identities has benefits. As Ray and Shank note, exploring and performing identity online granted them greater selfconfidence and self-esteem offline. In general, the well-being of the participants in this study increased as they were allowed to be themselves online, perhaps because they gained confidence in performing their identities in real life while knowing that a large support group waited for them online. This process can also, however, spur negativity. After noting the relationship between his alias and furry fandom, Rubi stated that the same occurs with internet trolls, or individuals that enjoy harassing, belittling, and bullying others online. He claimed that troll identity and politics, which was once a gag done solely online, has become more integrated with the identity of the person trolling. This may, in turn, affect the personalities and well-being of the troll. Trolling goes from being an act to a deviant identity that exists to bully and look down on others, often women. If rejected by mainstream society, they may then turn toward accepting deviant communities, like the alt-right or meninist groups. This could not be fully explored within the scope of this study and is based on the conjecture of a single participant. It should be, however, further explored in additional studies concerning internet subcultures.

\section{Multiple Identities and Belonging: Being a Furry and LGBT+}

Proponents of the dialogical-self refer to it as a diversity of voices, or identities, within an individual that converse with one another to create a sense of self (Owens, Robinson, and Smith-Lovin 2010). When an individual internalizes multiple deviant identities, these identities can be consciously viewed as separate aspects of a given person, instead of a descriptor of a whole. Logan, for example, describes their sense of self thusly:

Instead of being here, a beautiful tapestry of who I am and the things that make me, it's: I'm naked and I can put on clothing-various identities-on like a paper doll and I can take them off later or replace them. And who knows what I am when I have nothing on, but I can take on and take off things and whatever I'm wearing right now is what I am right now.

Regardless of how explicit Logan's statement is, it is evident throughout my conversations with interviewees that multiple, distinct identities based on their internalized or assigned deviance exist within them. As discussed in the 
literature review, furries can construct fantastic, alternate selves in the form of fursonas that can carry their own personalities. With his fursona, Rubi takes it a step further. He refers to his fursona as an entirely separate entity instead of an aspect of himself. He reflects that his fursona started as a version of his ideal self that then changed as his own confidence grew. He says, "I've given him some features, like personality traits and even a different voice than my actual voice," and he transforms into this alternate self when fursuiting: "there's a little bit of that magic where I'm becoming something else."

Ray's relationship with their fursona is similar, although the personality of their fursona is much more in line with Ray's own. They note, however, "he's [the fursona] not perfect and he's got flaws, reflective to my own but more pronounced, which I think helps me recognize and understand myself better." In this way, Ray's fursona is in dialog with other aspects of Ray's self, helping him grow as a person. It should also be noted that Ray refers to their fursona using he/him pronouns exclusively, but allows others to refer to themselves as they/them or he/him. This implies that Ray sees their fursona as a masculine ideal for themselves or as a more masculine version of themselves, which can affirm their identity or perhaps push them to achieve a specific form of gender embodiment.

But what happens with those identities? What happens when more than one nonnormative identity is accumulated by an individual? Based on both Owens, Robinson, and Smith-Lovin's (2010) and Lang and Lee's (2005) discussion of identity accumulation theory, I had assumed multiple identities would enable those with deviant identities to access multiple supportive spaces online: furry exclusive spaces, LGBT+ exclusive spaces, and the intersection of the two. This is not the case in the experience of my participants. Instead, the number of deviant identities reduces the number of online groups that participants interact with due to an intense experience of belonging within one all-encompassing, niche group: furry fandom. It is not that they cannot engage with multiple communities associated with their multiple identities; they choose not to because they have no need for those additional communities.

Among other identities that deviate from social norms, furry identity intersects strongly with LGBT+ identity. Through furry fandom, individuals are able to interact with similarly identifying people while consuming and producing content related to their niche interests, be those interests inherently furry or not. Unlike the members of LGBT+ exclusive communities that Acosta et al. (2014) and Downing (2013) studied, the LGBT+ furries that took part in this study did not have to or want to seek out exclusively LGBT+ spaces because online furry fandom is inherently LGBT+.

Several informants note that they did not know of many (or any) straight furries. There are so few cisgender straight furries that a relatively famous furry with whom I spoke casually about my research noted that he was the only cisgender straight furry he knew. This is an important aspect of furry fandom for the participants in this study. Terra, for example, states that they do not need other online communities because of the high LGBT+ population in furry fandom. They say, "It's just kind of like, they all get it. I don't have to explain what being non-binary is to someone. I don't have to explain what, like, they/them means to someone. It's just kind of, like, a prerequisite." Through furry fandom their need for belonging is largely satisfied.

Since the fandom online is so densely LGBT+, the content created and displayed through Show and Tell is also inherently LGBT+. It is not uncommon to see artwork of fursonas wearing pride merchandise, furries posting about their glowing love for their same-gender or trans partners, or intimate gift art for those same partners. Through Show and Tell, existing members of a deviant identity can create the means for others to further question their own identities, develop an interest in a new identity, or affirm existing identities. Logan notes that furry fandom is an accepting space for many deviant identities due to the fandom's own deviant nature. In this way, deviancy can beget deviancy because an individual has a secure sense of belonging in furry spaces to experience their multiple deviant identities. 
Several informants note that their exposure to and acceptance of their LGBT+ identity occurred through online furry fandom. Shank reflects on his experience accepting his bisexuality through furry fandom like this:

The furry community is largely comprised of diverse artists who promote each other but when you follow them [] you get a sneak peek into their personal life; often they share stories both good and bad about their experiences as LGBT+. So I feel I get to listen to the often unheard stories of LGBT+ which challenge common preconceptions/ assumptions that I held myself at one point or another.

Just as Downing (2013) found, interactions with the community, although passive in these cases, create a means of knowledge sharing that contributes to identity formation and increased well-being. For the participants, being a part of furry fandom allowed them to see that they were not alone and did not need to be alone in their queer identity.

\section{The Need to Belong and Improving Well-being}

Based on my initial understanding of the theories of dialogism and identity accumulation theory, I hypothesized that, by having multiple identities through accumulation and dialogism, one that identified as LGBT+ and as a furry would have access to LGBT+, furry, and LGBT+ furry communities online. Through access to these multiple communities, I thought that individuals would likely experience greater access to multiple pathways to social support and positive relationships, thereby improving their overall well-being. However, what I found instead was that the number of deviant identities actually reduces the number of online groups that participants interact with due to an intense experience of belonging within one allencompassing, niche group: furry fandom. They chose to engage online with this group because they have no need for additional niche groups to feel that they belong. In belonging to these spaces, I found that participants expressed a general improvement in their well-being.

LGBT+ furries of the past, through art, memes, videos, and snippets of their lives, created online spaces for LGBT+ furries of the present. On Twitter, this creates an accepting and affirming space and community. Before the internet, those who felt affiliated with deviant identities like LGBT+ or furry identity may not have had an accepting space in which to play those identities. As this study shows, this can have negative effects on individual well-being. Performing identities accepted by mainstream society in order to experience belonging when, in reality, that identity feels wrong can lead to distress, low self-esteem, and body image issues. In instances where participants did not pursue conformity with the mainstream, they lost social support from certain groups due to the stigma associated with deviance.

Through the production of LGBT+ furry media, furries online create community in which other budding furries can interact with others, learn about themselves, and unlearn stigma. Based on my conversations with my informants, having a community space online greatly improves well-being. The fandom gives members a place to belong and experiment with their identity in a relatively safe environment, allowing them to solidify their sense of self while improving their own creative skills and socializing. Online support also contributes to the embodiment of these identities in real life. Participants did not necessarily talk about their deviant identities with those outside of the community unless explicitly asked, but were confident enough not to hide their identities on or offline.

Deviant identities formed online became fully integrated into participants' sense of self and narrowed the number of communities they engaged with. This expands on the literature of identity accumulation theory, which generally emphasizes how multiple identities can either be in conflict with one another or give individuals access to multiple identity-based support systems (Lang and Lee 2005; Owens, Robinson, and Smith-Lovin 2010). This study finds, however, that there are other ways that multiple identities may work. LGBT+ and furry identities coexisted harmoniously rather than in conflict within individuals and reduced rather than increased the number of online communities they engaged with to one: furry fandom. This overall dynamic is regarded positively by the participants. Participants find 
no need to find other communities to belong to because furry fandom exists as a catch all. Every niche interest, every craft, every deviant identity exists within furry fandom, so there is no need to go elsewhere to feel like one belongs. Through this sense of community and belonging, the participants found themselves better off than before they joined the community.

Knowing how identity and belonging is experienced online is pertinent today because of the prevalence of the internet in the lives of those coming of age. Through studies like this, researchers can understand how the use of the internet affects people and culture. The worth of this study and other studies like it also rests in its humanizing element. Across the United States, LGBT+ identities are oppressed and furry identity is stigmatized, often due to misconceptions and misunderstandings. Studying people with these identities ethnographically can lift the veil of mystery around these identities, bringing awareness to the humanity of deviant identities. Anthropomorphizing the self is a very human practice, present throughout history in cave paintings and mythology. Through studies like this, we can humanize the stigmatized and further understand the colorful variance of human life. 


\section{Acknowledgements}

I wish to thank everyone who helped me craft this article. My supervisor Carolyn Schwarz, without whom I would have never considered publishing; the anthropology department of Goucher College, for giving me the tools to research and the curiosity to chase ideas down figurative and literal rabbit holes; my key informant, Logan, who supported me with invaluable info and cherished friendship; and, finally, the furries who disregarded all stranger-danger instincts and spoke with me about their experiences. 


\section{References}

Acosta, Leonor, Sebastian Molinillo, Esperanza Moreno, and Beatriz Gomez-Ortiz. 2017. "Alternative Sexualities and Virtual Communities: Aspects of LGBT Participation on Social Networking Sites in Spain." In Identity, Sexuality, and Relationships Among Emerging Adults in the Digital Age, edited by Michelle F. Wright, 75-97. Hershey, PA: IGI Global.

Atay, Ahmet. 2015. Globalization's Impact on Cultural Identity Formation: Queer Diasporic Males in Cyberspace. Lanham, MD: Lexington Books.

Bandlamudi, Lakshimi. 1994. “Dialogics of Understanding Self/ Culture." Ethos 22(4): 460-493.

Boellstorff, Tom. 2008. Coming of Age In Second Life: An Anthropologist Explores the Virtually Human. Princeton: Princeton University Press.

Brown, Meghan Ann. "Animal People." Master's thesis, Iowa State University, 2015.

Brubaker, Rogers, and Frederick Cooper. 2000. "Beyond 'Identity."' Theory and Society 29: 1-47.

Carlson, Marla. 2011. "Furry Cartography: Performing Species." Theater Journa/63(2): 191-208.

Chadborn, Daniel, Patrick Edwards, and Stephen Reysen. 2018. "Reexamining Differences Between Fandom and Local Sense of Community." Psychology of Popular Media Culture 7(3): 241-249.

Davis, Katie, and Emily Weinstein. 2017. "Identity Development in the Digital Age: An Eriksonian Perspective." In /dentity, Sexuality, and Relationships Among Emerging Adults in the Digital Age, edited by Michelle F. Wright, 1-17. Hershey, PA: IGI Global.

Downing, Gary. 2013. “Virtual Youth: Non-Heterosexual Young People's Use of the Internet to Negotiate their Identities and Socio-Sexual Relations." Children's Geographies 11(1): 44-58.

Gerbasi, Kathleen C., Nicholas Paolone, Justin Higner, Laura L. Scaletta, Penny L. Bernstein, Samuel Conway, and Adam Privitera. 2008. "Furries from A to Z (Anthropomorphism to Zoomorphism." Society and Animals 16: 197-222. 
Hatchel, Tyler J., Kaveri Subrahmanyam, and Michelle Birkett. 2017.

"The Digital Development of LGBTQ Youth: Identity, Sexuality, and Intimacy." In /dentity, Sexuality, and Relationships Among

Emerging Adults in the Digital Age, edited by Michelle F. Wright, 61-74. Hershey, PA: IGI Global.

Jeansonne, Sherry A. "Breaking Down Stereotypes: A Look At the Performance of Self-Identity Within the Furry Community." Master's thesis, Texas State University, 2012.

Lang, Josephine Chinying, and Chay Hoon Lee. 2005. “Identity Accumulation, Others' Acceptance, Job-Search Self Efficacy, and Stress." Journal of Organizational Behavior, 26(3): 293-312.

McNally, Victoria. 2014. "Let's Stop Making Jokes About Furries While Discussing That Recent Terrorist Attack on Furries." The Mary Sue, December 9, 2007. https://www.themarysue.com/furry-conterrorist-attack/.

Owens, Timothy J., Dawn T. Robinson, and Lynn Smith-Lovin. 2010. "Three Faces of Identity." Annual Review of Sociology 36: 477-99.

Půtová, Barbora. 2013. "Prehistoric Sorcerers and Postmodern Furries: Anthropological Point of View." International Journal of Sociology and Anthropology 5(7): 243-248.

Sampson, Edward E. 2008. Celebrating the Other: A Dialogic Account of Human Nature. Chagrin Falls, $\mathrm{OH}$ : Toas Institute.

Steiner, Peter. 1993. "On the Internet." The New Yorker, July 5, 1993. Available from https://www.newyorker.com.

Strike, Joe. 2017. Furry Nation: The True Story of America's Most Misunderstood Subculture. Jersey City, NJ: Cleis Press.

Tronstad, Ragnhild. 2008. "Character Identification in World of War Craft: The Relationship Between Capacity and Appearance." In Digital Culture, Play, and Identity: A World of Warcraft Reader, edited by $\mathrm{H}$. Corneliussen and J. Rettberg, 249-264. Cambridge, MA: MIT Press.

Van Meijl, Toon. 2010. "Anthropological Perspectives on Identity: From Sameness to Difference." In The Sage Handbook of Identities, edited by M. Wetherell and C.T. Mohanty, 63-81. Los Angeles, CA: Sage Press. 
Walks, Michelle. 2014. "'We're Here and We're Queer?': An Introduction to Studies in Queer Anthropology." Anthropologica 56(1): 13-16.

Wikipedia. 2020. "On the Internet, nobody knows you're a dog." Last modified April 24, 2020. https://en.wikipedia.org/wiki/ On_the_Internet,_nobody_knows_you\%27re_a_dog.

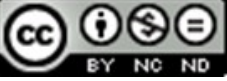

This work is licensed under a Creative Commons

Attribution-NonCommercialNoDerivatives

4.0 International License. 